\title{
Thalamic atrophy in frontotemporal dementia - not just a C9orf72 problem
}

Martina Bocchetta, Elizabeth Gordon, M. Jorge Cardoso, Sebastien Ourselin, Jason D. Warren, Jonathan D. Rohrer

Background: Frontotemporal dementia (FTD) is a heterogeneous neurodegenerative disorder associated with frontal and temporal atrophy. Subcortical involvement has been described too, with early thalamic atrophy associated with the C9orf72 expansion. We aimed to investigate the thalamic involvement in a large cohort of patients with FTD including those with genetic and pathological confirmation.

Methods: We investigated thalamic volumes in a sample of 315 FTD patients (age: mean(standard deviation) 64(9) years; disease duration: 7(13) years) compared with 84 age-matched controls (age: 62(12) years). We performed a parcellation of T1 MRIs using an atlas propagation and label fusion approach (Cardoso et al., 2015). Left and right thalamus volumes were combined and corrected for total intracranial volumes. We assessed subgroups stratified by clinical diagnosis (145 behavioural variant FTD (bvFTD), 79 semantic dementia (SD), 85 progressive nonfluent aphasia (PNFA), 6 with associated motor neurone disease (FTD-MND), genetic diagnosis (20 with MAPT, 20 with C9orf72, 8 with GRN mutations), and pathological diagnosis (39 tauopathy, 51 TDP-43opathy, 3 FUSopathy). We assessed the diagnostic accuracy based on thalamic volume.

Results: Overall, FTD patients had smaller thalami than controls ( $7 \%$ difference in volume, $\mathrm{p}<0.0005$, Mann-Whitney). Stratifying by genetics, C9orf72 group had the smallest thalami (16\% difference from controls, $\mathrm{p}<0.0005$ ) (Figure). However, the thalami were also smaller than controls in the other genetic groups: MAPT and GRN groups showed respectively an $8 \%$ and $10 \%$ difference $(\mathrm{p} \leq 0.001)$. The C9orf72 group had significantly smaller thalami than the MAPT group ( $8 \%$, $\mathrm{p}=0.011)$, but not the GRN group ( $\mathrm{p}=0.199$ ). ROC analysis showed a relatively poor ability to separate C9orf72 from MAPT $(\mathrm{AUC}=0.732)$ and from $G R N$ cases $(\mathrm{AUC}=0.719)$. All clinical subtypes had significantly smaller thalami than controls, with the FTD-MND group having the smallest (13\%, $\mathrm{p}=0.002)$, followed by bvFTD ( $8 \%, \mathrm{p}<0.0005)$, PNFA $(6 \%, \mathrm{p}<0.0005)$, and lastly SD $(5 \%, \mathrm{p}<0.0005)$. In the pathological groups, the TDP-43opathies had an $11 \%$ difference from controls $(\mathrm{p}<0.0005)$, and tauopathies $8 \%$ $(p<0.0005)$, while the FUSopathies did not show any difference from controls $(p=0.635)$. 
Conclusions: The thalamus was most affected in C9orf72 genetically, TDP-43opathies pathologically and FTD-MND clinically. However, thalamic atrophy is a common feature across all FTD groups, apart FUSopathies in which it seems relatively spared.

Figure.

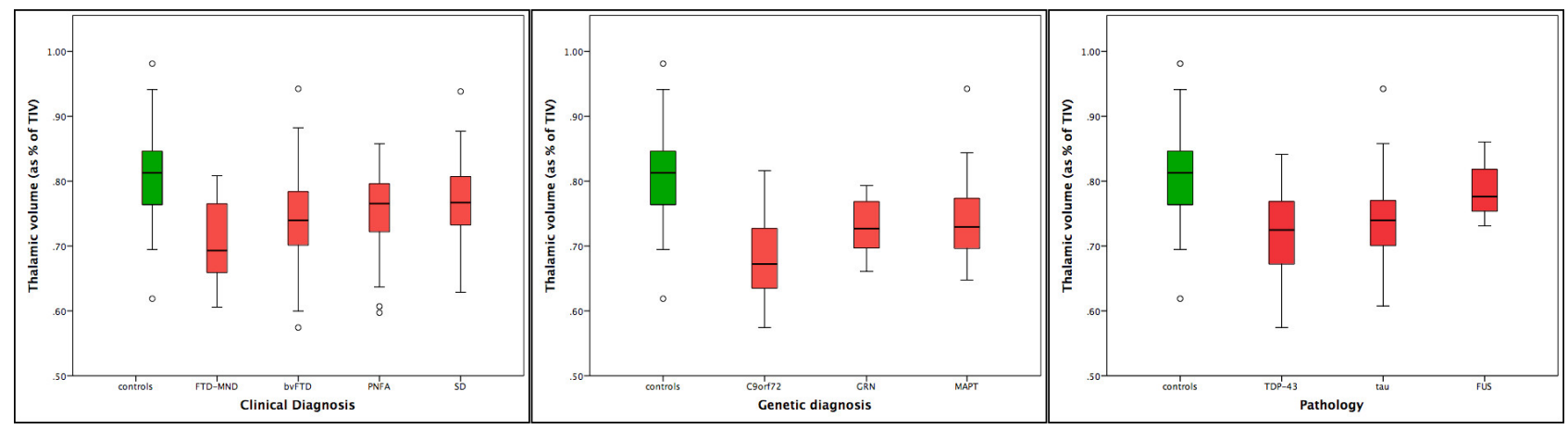

